\title{
Was uns das Gebet lehrt - Jean-Louis Chrétiens Phänomenologie des Gebets als Beitrag zu einer Hermeneutik des Gebets als leibliches Verstehen
}

Michael Ulrich Braunschweig

\section{Einleitung}

Das Gebet, darin dürften sich die Religionskundler ungeachtet ihrer disziplinären Beheimatung als Bindestrich-Soziologen, -Phänomenologen, -Historiker, -Philosophen und selbst die Theologen einig sein, darf als eines der grundlegenden Phänomene »des Religiösen« und vielleicht gar des Menschseins betrachtet werden. Es liegt auf der Hand: So zahlreich die verschiedenen Erscheinungsformen und die Kombinationsmöglichkeiten der konstitutiv oder nur statistisch mit dem Gebet korrelierenden Eigenschaften (laut oder still, öffentlich oder versteckt, individuell oder kollektiv, fragend, klagend, jubelnd, lobend, dankend, um nur wenige Aspekte zu nennen) bestimmt werden, so zahlreich sind auch die denkbaren Typologien, Wesensbestimmungen, Rückführungen und Interpretationen. Eine Auseinandersetzung, die das Gebet aber als Praxis bestimmter Lebensformen ernst nimmt, ohne es in seiner Authentizität oder Legitimität von vorneherein in Zweifel ziehen zu wollen, wird m.E. allerdings nicht umhinkommen, über die reine Konstatierung seines empirischen Vorkommens und eine im Äußerlichen verbleibende Beschreibung seiner Erscheinungsformen hinauszugehen und sich durch die vom Phänomen selbst aufgeworfenen Fragen herausfordern lassen. Kurz: Man wird sich auf das die Hermeneutik des Gebets durchaus im subjektiven Sinne dieses Genitivs einzulassen haben und sich belehren lassen, welches Verständnis von Leben und Menschsein das Gebet und das Beten selbst lehrt, vermittelt und zu verstehen aufgibt. Die folgenden Ausführungen mögen als Beitrag zu Anliegen verstanden werden im Rekurs auf den an der Sorbonne lehrenden Philosophen Jean-Louis Chrétien (“1952), der zu den profilierten Vertretern der jüngeren französischen Phänomenologie gerechnet werden darf.

Obgleich er bereits 1991 von Dominique Janicaud in seinem die Diskussion um eine "theologische Wende« in der Phänomenologie 
eröffnenden Büchlein ${ }^{1}$ sowie von Jean-François Courtine $1992^{2}$ zu den führenden Vertretern einer neuen Richtung in der Phänomenologie gerechnet wurde, die auf innovative Weise die Grenzen der traditionellen Phänomenologie im Hinblick auf die Möglichkeit einer Phänomenologie der Religion bzw. der religiösen Erfahrung ausloteten, ist er im deutschen Sprachraum noch immer weitgehend unbekannt. ${ }^{3}$ Dieser Unbekanntheit ein kleines Stück entgegenzuwirken mag deshalb als Nebenintention dieses kurzen Beitrags wahrgenommen werden. Dies lässt es geboten erscheinen, der Stimme Chrétiens selber gebührlichen Raum zuzugestehen. So nehme ich mir in diesem Artikel die Aufgabe heraus, in eher darstellender Weise Chrétiens Phänomenologie des Gebets in ihren grundlegenden Dimensionen und im Hinblick auf ihre Dialogfähigkeit mit der Theologie herauszuarbeiten.

\section{Einleitende Bemerkungen zu Jean-Louis Chrétien}

In einem in der Zeitschrift Critique erschienen Interview aus dem Jahr 2012 nennt Jean-Louis Chrétien, in dessen Werken man, abgesehen von einem Vorwort in der zweiten Auflage von L'inoubliable et l'inespérét, explizite Hinweise zu Methodik, Arbeitsweise und inhaltlichem Zentrum eher vermissen mag, als übergreifendes Thema bzw. »roten Faden" seiner phänomenologischen Arbeiten eine "Phänomenologie der Sprache (parole) als Ort, an dem jeglicher Sinn zu Tage tritt und sich sammelt. Diese Sprache ist diejenige der Endlichkeit, da sogar das Wort Gottes (parole de Dieu) uns nur in denen des Menschen zukommt. $\|^{5}$ Die Sprache führt uns dabei an

\footnotetext{
1 Dominique Janicaud, Le tournant théologique de la phénoménologie française, Combas 1991, rechnet Jean-Louis Chrétien neben Emmanuel Lévinas, Michel Henry und Jean-Luc Marion zu den führenden Vertretern der neuen Richtung der Phänomenologie, vgl. 20 u. 51-54.

2 Jean-François Courtine, Phénoménologie et Théologie, Paris 1992, stellt Chrétien in eine Reihe mit so bekannten wie profilierten Vertretern einer Religionsphänomenologie wie Paul Ricœur, Michel Henry und Jean-Luc Marion.

3 Eine Ausnahme bildet die jüngste Überblicksdarstellung Hans-Dieter Gondek/ László Tengelyi, Neue Phänomenologie in Frankreich, Frankfurt a.M. 2011, darin insbesondere 569-580. Im früheren von Bernhard Waldenfels stammenden Bericht (Phänomenologie in Frankreich, Frankfurt a.M. 1983) ist von Chrétien wie von der ganzen "jüngeren" Generation noch nicht die Rede.

4 Jean-Louis Chrétien, L'inoubliable et l'inespéré, Paris 1991.

5 Jean-Louis Chrétien, "Essayer de penser au-delà de la subjectivité«. Entretien réalisé par Marc Cerisuelo et Camille Riquier, in: Critique 790 (2013), 241-253, 243
} 
Grenzphänomene des Daseins, denen Chrétien in seinen Schriften nachspürt. Dabei beschränkt Chrétien sich aber nicht bloß auf eine, in ihrer phänomenologischen Möglichkeit ohnehin äußerst zweifelhafte ${ }^{6}$, Begegnung mit dem Bereich des Religiösen unter seinen unterschiedlichen Namensgebungen (dem Heiligen, dem Göttlichen, dem Absoluten u.v.a.m.). Seine seit Mitte der 80er Jahre im jährlichen Rhythmus erscheinenden Studien sind als Antwortversuche auf seine ins Existenzielle gewendete phänomenologische Grundfrage wahrzunehmen: "Wie [soll man] auf das antworten, was gemessen an all unseren Möglichkeiten in der Begegnung mit der Welt regelrecht überschießt und sich nicht einordnen lässt? « ${ }^{7}$ Dem Ziel einer solchen Phänomenologie nähert sich Chrétien in einer Reihe von Analysen von »Begegnungen" (rencontres) mit Phänomenen des Überschusses (excès) wie dem Schönen ${ }^{8}$, dem Versprechen ${ }^{9}$, aber auch Gegenstandsbereichen, die sich nur mehr an den Grenzen phänomenaler Erscheinung erweisen, wie etwa der Frage nach einem Bereich des Unvergesslichen im Vergessen ${ }^{10}$ oder des Unhörbaren im Hören ${ }^{11}$. Die Wortwahl der "Begegnung" (rencontres) soll dabei anzeigen, dass "die Initiative hier den Phänomenen selbst überlassen wird « ${ }^{12}$. Es ist bezeichnend für Chrétiens Arbeitsweise, dass die Analyse dieser Begegnungen sich nie auf Erfahrungen der Unmittelbarkeit berufen, sondern immer vermittelt im Gespräch und in der Auseinandersetzung nicht nur mit der philosophischen, sondern gerade auch der religiösen oder theologischen Tradition stattfindet. Disziplinäre Grenzen interessieren ihn dabei wenig - seine Hinwendung zur religiösen Tradition wie z.B. seine auch theologisch gehaltvollen Interpretationen biblischer Texte ${ }^{13}$ verfolgen vielmehr die Absicht, die philosophische Fruchtbarkeit des biblischen Glaubens jenseits falscher oder vermeintlicher Evidenzen aufzuzeigen

(hier wie im Folgenden, wenn nicht anders angegeben, jeweils eigene Übersetzungen, MUB).

${ }^{6}$ Vgl. dazu grundsätzlich Bernhard Waldenfels, Phänomenologie der Erfahrung und das Dilemma der Religionsphänomenologie, in:Wolf-Eckart Failling u.a. (Hg.), Religion als Phänomen. Sozialwissenschaftliche, theologische und philosophische Erkundungen in der Lebenswelt, Berlin/New York 2001, 63-84.

7 Chrétien, Entretien, 243.

8 Jean-Louis Chrétien, L'effroi du beau, Paris 1987.

9 Jean-Louis Chrétien, La Voix nue: phénoménologie de la promesse, Paris 1990.

${ }^{10}$ Chrétien, L'inoubliable et l'inespéré.

${ }^{11}$ Jean-Louis Chrétien, L'arche de la parole, Paris 1998.

${ }_{12}$ Gondek/Tengelyi, Neue Phänomenologie, 570.

${ }_{13}$ Z.B. in Jean-Louis Chrétien, Sous le regard de la Bible, Montrouge Cedex 2008. 
und dadurch neue Fragehorizonte zu öffnen. ${ }^{14}$ Im Rekurs auf poetische, biblische und philosophische Autoren versucht er dabei Dimensionen der Erfahrung zu erschließen, die gemeinhin verdeckt bleiben, sich phänomenologischer Beschreibung zu entziehen scheinen oder an den Extremen von Erfahrung und Denken anzusiedeln sind, um, ohne Anspruch auf eine kohärente Systemarchitektur, eine Phänomenologie rauf dem Weg auszuarbeiten, in der die zentrale menschliche Erfahrung des Mysteriums seinen Platz haben kann. ${ }^{15}$

\section{Chrétiens Phänomenologie des Gebets}

In diesem Zusammenhang kommt auch seine Auseinandersetzung mit dem Gebet zu stehen. Dabei sind vorderhand zwei wesentliche Elemente hervorzuheben: zunächst die Auffassung, dass jede Sprechhandlung (acte de parole) ${ }^{16}$ immer responsorischen Charakter hat, indem sie, selbst im Monolog, immer anderen, der Welt oder auch Gott antwortet. Damit verbunden ist das zweite von Chrétien betonte Moment, wonach Rede wesentlich leibhaften Charakter hat, insofern sie an einen menschlichen Körper und seine Stimme gebunden ist. Diese zwei Momente sind auch bestimmend für seine Analyse des Gebetsphänomens, der ich mich nun zuwenden will. Chrétien lässt keinen Zweifel an der herausgehobenen Bedeutung des Gebets für jede Beschäftigung mit Religion:

"Das Gebet ist das religiöse Phänomen par excellence, denn es ist die menschliche Handlung, die als einzige die religiöse Dimension öffnet und niemals aufhört, deren Öffnung zu erhalten, zu ertragen und darunter zu leiden. [...] Mit dem Gebet erscheint und verschwindet das Religiöse. ${ }^{17}$

\footnotetext{
${ }^{14}$ Vgl. Chrétien, Entretien, 246.

15 Vgl. Anne A. Davenport, Translator's Preface, in: Chrétien: The Call and the Response, vii-xxix.

${ }^{16}$ Es sei darauf hingewiesen, dass der in diesem Zusammenhang zentrale und von mir als "Sprechhandlung übersetzte Terminus eines "acte de parole« mangels entsprechender Referenzen in seinen Texten wohl e silentio als genuine Kategorie Chrétiens anzusehen ist und weder von John Searles Speech Acts (Cambridge 1969) abhängt (vgl. die frz. Übersetzung von Hélène Pauchard, Les actes de langage, Paris 1972) noch auf der Unterscheidung von parole, langue und langage aus der linguistischen Theorie Ferdinand de Saussures aufruht.

17 Jean-Louis Chrétien, La parole blessée. Phénoménologie de la prière, in: Courtine: Phénoménologie et Théologie, 1992, 41-78, 41.
} 
Die Grundzüge von Chrétiens Phänomenologie des Gebets ${ }^{18}$ sind rasch skizziert: ${ }^{19}$

1. Beten ist eine Sprechhandlung und entsprechend der beiden wesentlichen Bestimmungen von Sprache zugleich als Antwortphänomen zu verstehen und an die grundlegende Leiblichkeit der Sprache, die Stimme, konstitutiv verwiesen.

2. Alle Erscheinungsformen von Gebeten (seien es Dank-, Bitt-, Buß- oder Klagegebete) sind, selbst wenn sie stumm erfolgen, letztlich vom stimmhaften Gebet abhängig.

3. Das stimmhafte Gebet als solches ist wesentlich dadurch bestimmt, dass der oder die Betende sich selbst gegenüber einem unsichtbaren Anderen präsentiert.

4. Wenn das stimmhafte Gebet dabei nicht zu einem reinen Selbstgespräch zusammenschrumpfen soll, so ist es als "Exposition" gegenüber diesem Anderen zu konzipieren.

5. Stimmhaftes Gebet erweist sich weiter als agonales Transformationsphänomen, indem es uns von unserer Ego-Zentriertheit radikal von uns weg orientiert.

Die ersten beiden Bestimmungen folgen aus der Auffassung von Sprache als primär gesprochene Sprache bzw. Sprechen, das auf entsprechende physiologische Produktionsorgane angewiesen ist. Wie sich die übrigen Bestimmungen daraus ergeben, versuche ich im Folgenden zu rekonstruieren.

\section{Die Bedeutung des Redecharakters des Gebets}

Anders als Kant, der dem Gebet nur seinen "Geist", »die alle unsere Handlungen begleitende Gesinnung, [...] als ob sie im Dienste Gottes geschehen " abgewinnen konnte und insofern in allen sprachlich artikulierten Erscheinungsformen von Gebeten »höchstens nur den Wert eines Mittels zu wiederholter Belebung jener Gesinnung in uns selbst $\aleph^{20}$ zuerkennen wollte, geht Chrétien nicht von der Frage der moralpädagogischen Nützlichkeit des Gebets als charakterformender Disziplin aus, sondern fragt nach den dem Gebetsvollzug

\footnotetext{
${ }^{18}$ Ich beziehe mich hier auf den zuerst bei Courtine, Phénoménologie et Théologie, 1992, 41-78, erschienenen und später in L'arche de la parole, Paris 1998, 23-54, wieder abgedruckten Aufsatz La parole blessée. Phénoménologie de la prière.

${ }^{19}$ Vgl. ähnlich auch Anne A. Davenport, Translator's preface, in: Chrétien, The Call and the Response, vii-xxix, ix.

${ }^{20}$ Immanuel Kant, Die Religion innerhalb der Grenzen der bloßen Vernunft, AA VI, $195 f$.
} 
vorausgehenden und den durch das Gebet implizierten und bewirkten Veränderungen des Betenden.

Sehr allgemein diagnostiziert er zunächst eine mit dem Gebet in den verschiedensten Traditionen verbundene Überzeugung: "[...] nur beim Beten kann man Gott zugewandt sein und nur Gott zugewandt kann man beten. $\|^{21}$ Dabei stellt sich der phänomenologischen Beschreibung freilich ein doppeltes Problem. Denn "für die Konstitution der Bedeutung des Gebets [ist] der Adressat teilweise wesentlich. ${ }^{22}$ So scheint für den Betenden einerseits die Existenz des Adressaten eine unabdingbar notwendige Voraussetzung zu sein und andererseits scheint die Art und Weise der im Gebet vollzogenen Zuwendung, die Namen der Anrufung, die vorbereitenden Rituale, die vorgeschriebenen Formeln und als angemessen erachteten Körperhaltungen usw. von konstitutiver Bedeutung für die phänomenologische Beschreibung und damit die Möglichkeit einer Phänomenologie des Gebets selbst zu sein. Um der phänomenologisch nicht einholbaren onto(theo)logischen Setzung eines göttlichen Gegenübers im Gebet sowie der durch kulturelle und historische Kontingenz bedingten Pluralität von Erscheinungsformen zu entgehen, beschränkt Chrétien sich darauf, das Gebet als Sprechhandlung (acte de parole) anzusehen und danach zu fragen, "warum und wie wir im Gebet Stimme verleihen" und was dabei "der Sinn der verschiedenen Formen des Vortrags « ${ }^{23}$ ist.

Eine erste Beschreibung kennzeichnet das Gebet sodann als "Akt der Gegenwart gegenüber dem Unsichtbaren «: »Dieser Akt der Gegenwart setzt den ganzen Menschen aufs Spiel, in allen Dimensionen seines Seins, er exponiert ihn in jeglichem Sinn dieses Begriffs ${ }^{24}$. Diese Selbstpräsentation kann aber nur vollkommen sein und die ganze Existenz ins Spiel bringen, wenn sie nicht rein geistiger Natur ist, sondern auch die leibliche oder körperliche Existenz umfasst, wie Chrétien gegen Kant einwendet, was nur der Fall sein kann, wenn das Gebet selbst in diesem Sinne konstitutiv in der Leiblichkeit der Sprache "inkarniert" ist und nicht allein im Vernunftpostulat eines Gottesgedankens seinen Ort hat. Dies ist die erste Pointe der oben genannten Entscheidung, das Gebet unter dem Gesichtspunkt seiner Sprachlichkeit anzusehen. Bitten, fragen, klagen, loben, danken und was sonst noch die modalen Erscheinungsformen von Gebetsvollzü-

\footnotetext{
${ }^{21}$ Chrétien, La parole blessée. Phénoménologie de la prière, in: Courtine, Phénoménologie et Théologie, 1992, 41-78, 41.

22 Ebd., 43.

${ }^{23}$ Ebd., 44.

${ }^{24}$ Ebd., 45.
} 
gen sein mögen, sind, sofern sie nicht in der sprachlosen Phonalität bloßen Schreiens und Stöhnens verbleiben, sondern eben als Fragen nach ..., als Klagen über ... usw. immer auch einen intentionalen und propositionalen Gehalt artikulieren, per definitionem sprachlich verfasst. ${ }^{25}$

Ein zweites Moment kommt mit der Ausrichtung von Sprachvorgängen ins Spiel, insofern sie sich an jemand anderen richten. Denn gerade indem wir uns in jeder Anrede, nicht nur im Gebet, an einen anderen richten, uns ihm zuwenden, finden wir uns selbst durch diesen Vorgang in bestimmter Weise ausgerichtet, orientiert und affiziert:

"Die Rede (parole) affiziert und verändert den Absender und nicht den Adressaten. Wir affizieren uns selber vor dem anderen und zu ihm hin. Dies ist die erste Wunde der Rede (parole) im Gebet: [...] Ein anderer ist still in den Dialog mit mir selbst eingedrungen und hat ihn radikal verändert und gebrochen. $\otimes^{26}$

Es wird deutlich, dass dem Rede-Paradigma hier gegenüber anderen Kommunikationsvorgängen prioritären Charakter zukommt: Die reale Gegenwart des Gegenübers verändert die Art und Weise der Rede in ganz anderer Weise als dies in Kommunikationssituationen der Fall ist, in denen der andere lediglich virtuell oder imaginär vorhanden ist wie in allen Fällen von schriftlich vermittelter Kommunikation. Doch ergibt sich für die Beschreibung des Gebets als eines kommunikativen Vorgangs eine Problematik: Denn während in interpersonalen Beziehungen Fragen nach Art und Ausmaß von Symmetrie, Wechselseitigkeit und Dialogizität solcher Kommunikationsvorgänge phänomenologisch erhoben werden können, verschließt sich das Gebet dieser Beschreibungsperspektive in gewissem Sinn, weshalb ein an einem objektivierenden Kommunikationsschema von Absender-Botschaft-Adressat orientiertes Analysemodell dem sprachlichen Geschehen, das das Gebet ausmacht, nicht gerecht wird. Die Beschreibung hat sich vielmehr allein am Betenden zu orientieren, wobei der für den Betenden selber womöglich als real präsent geltende Gegenüber im Horizont der phänomenologischen Beschreibung "lediglich" virtuell als hypothetisches Zugeständnis einer weder veri- noch falsifizierbaren Möglichkeit in den Blick

\footnotetext{
${ }^{25}$ Schriftliche fixierte Gebete wie die Psalmen oder Gebetsformulare sind insofern reiner Text und werden erst durch die Aneignung in der Rede des Betenden zum Gebet, vgl. Chrétien, La parole blessée, 74.

${ }^{26}$ Ebd., 48 f.
} 
kommt, die aber nichtsdestotrotz ihre auf den Betenden zurückwirkenden Effekte entfaltet, was bei Chrétien sich wie folgt ausnimmt:

"Gott bitten, durch die Rede (parole) eine Fragehandlung zu vollführen, heißt, mit ihm sprechend, gleichzeitig und untrennbar etwas von ihm und etwas von uns zu sagen. Wir manifestieren uns gegenüber uns selbst, durch die Rede (parole) werden wir gegenüber uns selber manifest, während wir uns ihm gegenüber manifestieren. Bitten heißt handelnd anzuerkennen, nicht der Ursprung alles Guten und aller Gabe zu sein, und handelnd denjenigen anzuerkennen, an den wir uns um dessen willen wenden, was er ist. $\ll^{27}$

Insofern jedes Gebet Gott als Geber des Guten bekennt, entwindet es uns unserer Selbstbezüglichkeit. So findet sich der Betende im Gebet selber verändert wieder und diese Veränderung ist nach Chrétien eine weitere wesentliche Bestimmung des Gebets. Im Gebet selber als der Antwort auf einen den Betenden immer uneinholbar übersteigenden und ihm vorausgehenden Ruf, lernt der Betende, dass er nicht beten kann. ${ }^{28} \mathrm{Ob}$ das Gebet eine Veränderung bei Gott intendiert oder nicht, ist in dieser Hinsicht nicht entscheidend, jedoch die in diesem Ringen um das Gebet, das er nicht beten kann, dem Betenden widerfahrende Veränderung. So kann Chrétien als inhärentes Telos des Gebets beschreiben: "Das Gebet bittet darum, dass die Bitte nach dem Erbetenen unterbrochen werde, denn indem es bittet, hat es erhalten "29. So erweist sich der Kampf des Betenden im Gebet nicht nur als Kampf um das Beten-Können, sondern als Kampf "wirklich zu lernen, danke zu sagen " $^{30}$ : „Der Betende betet, um beten zu können und zunächst, um zu lernen, dass er es nicht kann, und er ist dankbar für sein Gebet wie für eine Gabe Gottes. ॥1 $^{31}$

\footnotetext{
${ }^{27}$ Ebd., 49.

${ }^{28}$ Vgl. ebd., 54f.

${ }^{29}$ Jean-Louis Chrétien, La prière selon Kierkegaard, in: Kairos 10 (1997), 131-140, 136.

${ }^{30}$ Chrétien, La prière selon Kierkegaard, 137.

${ }^{31}$ Chrétien, La parole blessée, 54.
} 


\section{Der Gebetscharakter der Sprache und seine ethi- schen Implikate}

Die Sprachlichkeit des Gebets bringt es mit sich, dass das Gebet, selbst wenn es einsam erfolgt, niemals losgelöst von seiner konstitutiven Einbindung in einen sozialen Kontext betrachtet werden kann. Dabei ist für Chrétien allerdings nicht nur die genetische Abhängigkeit von einer Sprachgemeinschaft im Blick, sondern die menschliche Stimme als »Ort der Rückkehr von der Welt zu Gott « ${ }^{32}$ und ihre unvertretbare Aufgabe im Horizont der ganzen Schöpfung. Nun gehört es zur Originalität von Chrétiens Gebetshermeneutik, dass die Frage des Verhältnisses von Sprache und Gebet sich nicht bloß darauf reduziert, dass jedes Gebet konstitutiv auf Sprache verwiesen ist, sondern auch umgekehrt gerade die Sprache wesentlich durch das Gebet bestimmt ist, ja wesentlich Gebetscharakter hat.

Diese These ist nun alles andere als selbstverständlich. Sie gründet in Chrétiens Auffassung von der Sprache als einer Arche derWelt: Mit Rekurs auf die Schöpfungsgeschichte der Genesis entfaltet Chrétien, wie der erste Sprachgebrauch Adams nicht in der Informationsvermittlung, sondern in der Benennung der Welt besteht. Die Sprache überreicht uns die Welt und verleiht den Phänomenen Stimme. ${ }^{33}$ Dabei rekurriert Chrétien auf einen Gedanken von Lévinas, wonach »thematisieren heißt, dem Anderen die Welt durch das Wort an[zu] bieten $\aleph^{34}$ :

"Die dem Menschen eigentümlichste Rede (parole) ist die zum anderen hingewendete Rede (parole), dem anderen gegeben, eine Rede von Übersiedlung und Überschreitung, eine exzentrische Rede.Was zu uns gehört ist, eine Rede äußern zu können, die aufhört, sich selbst zu gehören. Sie ist wie die Liebe nicht substituierbar, ohne vorstellbare Stellvertretung; kein anderes Werk kann diese Rede ersetzen und nichts kann uns in dieser Rede ersetzen. $\aleph^{35}$

Die Rede zeichnet den Menschen unter den Geschöpfen Gottes aus. Nach dem Schöpfungsbericht ist sie die den Menschen auszeichnende Gabe Gottes. Tiere können mit ihren Organen zwar

\footnotetext{
${ }^{32}$ Ebd., 76.

${ }^{33}$ Vgl. Chrétien, L'arche de la parole, $156 f$.

${ }^{34}$ Emmanuel Lévinas, Totalité et Infinie. Essai sur l'extériorité, Den Haag 1961, 74; dt. Totalität und Endlichkeit. Versuch über die Exteriorität, übers. v. Wolfgang Nikolaus Krewani, Freiburg/München 1987, 104.

${ }^{35}$ Chrétien, L'arche de la parole, 167.
} 
Laute produzieren, diese unterscheiden sich aber neben vielen anderen Aspekten von der menschlichen Sprache, insofern Letztere nicht in ihrer unmittelbaren Präsenz aufgeht, sondern gerade auch das gegenwärtig machen kann, was längst vergangen, weit entfernt oder noch nicht erreicht ist. Engel auf der anderen Seite sind als körperlose Geistwesen nicht fähig, den Dingen und der Welt Stimme zu verleihen, denn als rein geistige Wesen verfügen sie nicht über die entsprechenden Fähigkeiten stimmlicher Artikulation ${ }^{36}$, die den Menschen auszeichnet. Die angemessene Antwort auf die Sprache als Gabe Gottes sieht Chrétien in den Sprachformen der Darbringung (offrande) und des Lobs (louange): In ihm [i.e. dem Lob, MUB] wird die Gabe empfangen und können wir etwas geben, das uns gehört, das heißt uns. $\|^{37}$

Das "Dankbarsein-Können« als Zielbestimmung der Sprache darf nun nicht als enthusiastische oder sentimentale Verkürzung oder supralapsarische Verharmlosung einer selten zu Dankbarkeit Anlass bietenden Wirklichkeit missverstanden werden: "[J]eder weiß, dass das Lob des Brotes die Hungrigen nie gestillt hat und ihren Hunger nur noch schlimmer machen kann. $\aleph^{38}$ Aber gerade darin zeigt sich die ethische Dimension der Darbringung (offrande) der Welt in der Sprache. Sie verlangt, dass das Dargebrachte der Gabe angemessen ist - und "nur Leben kann gegeben werden ${ }^{39}$ :

"Die Bejahung ist der einzige Ort des Kampfes mit dem Bösen. Sagt man Nein auf das Nein, so sagt man doch immer nur Nein [...]. [...] einzig der Diamant des Ja vermag jede Verneinung dauerhaft, in ihrem Herzen, zu bekämpfen, ohne sie leugnen zu müssen. « $^{40}$

Insofern der Mensch das im Gegensatz zu Tieren und Engeln mit einer stimmhaften Sprache begabte Wesen ist, kommt ihm im Modus des Zeugnisses eine unvertretbare und unersetzbar geforderte Aufgabe zu: In der Sprache des Menschen kommt auch die sprachlose Welt zur Sprache, im Lob der Schöpfung wird auch die ganze sprachlose Kreatur Gott - dem Geber der Sprache - entgegenge-

\footnotetext{
36 Die metaphorische Rede von "Engelsstimmen« erweist sich dabei als Oxymoron und verweist damit auf das phänomenologische Grenzproblem, dass der Ruf oder Anspruch Gottes oder von Engeln sich phänomenologischer Beschreibung wesentlich entzieht, was sich sprachlich in oxymorotischen oder paradoxen Wendungen niederschlägt.

${ }^{37}$ Chrétien, L'arche de la parole, 167.

${ }^{38}$ Ebd., 173.

${ }^{39}$ Ebd.

${ }^{40}$ Ebd., 199.
} 
bracht. Darin besteht dieVerantwortung des Menschen für die ganze Schöpfung.

\section{Theologie oder Phänomenologie? Einige kritische Anfragen an Chrétien}

Man mag auf diese eucharistische Deutung menschlicher Sprache mit einer gewissen Reserve reagieren. Aus ethischer Perspektive mag man etwa darauf verweisen, dass Chrétiens Interpretation auf einer $\mathrm{zu}$ optimistischen inkarnationstheologischen Auffassung menschlicher Möglichkeiten beruht: Mit der Deutung von Sprache, die in Lob und dankbarer Darbringung zu ihrem Ziel kommt, wird zwar der individuellen Unvertretbarkeit Rechnung getragen und kommt die menschliche Verantwortung für soziale, ökonomische, politische und auch ökologische Verhältnisse als notwendiger Voraussetzung individuellen Lobs und Dankes zum Tragen. Doch scheint gerade die Ambivalenz der Bedeutung von Sprache dort unterbelichtet zu sein, wo die Frage danach, was nun gerade nicht zur Sprache kommt, virulent und ethisch problematisch ist ${ }^{41}$ In menschlicher Rede kommt nie alles zur Sprache und gerade das Unaussprechliche, Unausgesprochene, Verschwiegene und Unterdrückte sowie die Realität von Subalternitätsverhältnissen verweisen auf die prekäre Ambivalenz einer einseitig an den eucharistischen Möglichkeiten orientierten Auffassung menschlicher Sprache als inkarnatorisch ins Recht gesetztem (Herrschafts-)Instrument.

In philosophischer Perspektive scheint mir zudem der Hinweis, dass die Chrétiens Auffassung zugrundeliegende Annahme eines göttlichen Ja zur guten Schöpfung ein Gedanke ist, den "die Philosophie als solche nicht aussprechen kann ${ }^{42}$, auf ein methodologisches Problem hinzuweisen, das ich im Folgenden kurz mit Bezug auf Paul Ricœur zu umreißen versuche. Bei aller Anerkennung der Eigenständigkeit von Chrétiens wohlwollender Zuwendung zu Traditionsbeständen religiöser Literatur und des Muts, den ein solches

\footnotetext{
${ }^{41}$ Vgl. dazu Judith Butler, Excitable Speech. A Politics of the Performance, London und New York 1997.

${ }^{42}$ Gondek/Tengelyi, Neue Phänomenologie, 580. Davon abweichend und grundsätzlich Chrétiens Ansatz als wertvollen Beitrag zu einer von methodischen Selbstverboten befreiten Phänomenologie zustimmend aufnehmend jüngst: Rolf Kühn, Französische Religionsphilosophie und -phänomenologie der Gegenwart. Metaphysische und post-metaphysische Positionen zur Erfahrungs(un)möglichkeit Gottes, Freiburg u.a. 2013, 237-246, insb. 240, Anm. 123.
} 
Unterfangen wohl braucht im derart stark von Laizismus geprägten Milieu der Pariser Großstadt, wird man nicht umhin kommen, die Frage nach der >Unterscheidung der Geister und möglicher Kritik zu stellen.

In methodologischer Hinsicht stellte bereits der 1992 von JeanFrançois Courtine herausgegebene Band Phénoménologie et Théologie die Differenzen von Chrétiens Ansatz gegenüber denjenigen von Jean-Luc Marion, Michel Henry, aber auch Paul Ricœur heraus. Während erstere darauf abzielen, in Auseinandersetzung mit der religiösen Tradition bzw. religiösen Phänomenen eine radikale Transformation der Phänomenologie selbst herbeizuführen, die wie im Falle Henrys selbst vor einem spinozistischen Ineinanderfallen von Leben und Gott nicht zurückschreckt, sondern darin gerade das Ziel einer radikalen Lebenshermeneutik sieht, verwies Ricœur mit deutlicher Reserve auf das grundlegende Dilemma einer Phänomenologie der Religion: den Umstand, dass man zwar wohl die Existenz religiöser Gefühle behaupten kann, dass diese aber nie unmittelbar gegeben sind, sondern nur vermittelt durch Sprache, Kultur und Geschichte und deshalb das Vorhaben einer Phänomenologie der Religion, zu einer universellen Bestimmung des religiösen Phänomens bzw. der Religion zu gelangen, sich mit einer unermesslichen Pluralität von Phänomenen konfrontiert sieht, deren Rückführung auf eine allgemeine, geschweige denn anthropologische, Struktur ${ }^{43}$ auf theoretisch nicht weiter begründbaren und auch nicht von den Phänomenen selbst her sich aufdrängenden Voraussetzungen beruht, die damit letztlich per petitionem principii festlegen, was die phänomenologische Analyse zutage fördern sollte. ${ }^{44}$ Selbst die, auch von Chrétien in Anspruch genommene, Annahme einer religiöser Grundstruktur von Ruf und Antwort ("appel-réponse«) sei nie unabhängig von den unterschiedlichen historischen Wirkungen gegeben, in denen diese Struktur allenfalls inkarniert ist, und entsprechend ungesichert ist auch die Möglichkeit, sie als universale Wesenseigenschaft von Religion ausweisen zu können. ${ }^{45}$ Dies hat für Ricœur die entscheidende Konsequenz, dass das Ansinnen einer Phänomenologie eines universalen religiösen Phänomens zurückgewiesen werden muss und man sich damit zu begnügen habe, die Grundlinien einer bestimmten religiösen Tradition hermeneutisch aufzuweisen. ${ }^{46}$ Jeder über diese

\footnotetext{
${ }^{43}$ Vgl. Paul Ricœur, Expérience et langage dans le discours religieux, in: Courtine, Phénoménologie et Théologie, 1992, 15-39, 19.

${ }^{44} \mathrm{Vgl}$. Ricœur, Expérience et langage, 21.

${ }^{45}$ Vgl. ebd., 19.

46 Vgl. ebd., 20.
} 
religionsinterne Hermeneutik hinausgehende Versuch einer universalen Phänomenologie des religiösen Phänomens kann methodologisch nur als sekundäre Ausweitung und mithilfe von Übersetzungsprozeduren erfolgen, deren Plausibilitätspotential letztlich immer auf dem dabei in Anspruch genommenen Analogieverfahren beruht. ${ }^{47}$

Nimmt man diese Bedenken Ricœurs ernst, so scheint mir, müsste man sich mit einer an einer partikularen Tradition orientierten Hermeneutik des Gebets begnügen, ohne sie in einen phänomenologisch vermeintlich ausweisbaren Bereich des anthropologisch Allgemeinen erheben zu wollen. Dabei käme man aber nicht umhin, sich auch mit Fragen der traditionsinternen Gebetskritik auseinanderzusetzen: Man kann selbstredend zu Vielem beten und allem Möglichen »Opfer darbringen" - Chrétien selbst gesteht, wie gesehen $^{48}, \mathrm{zu}$, dass der Adressat des Gebets für die Art und Weise von Anbetung und Darbringung konstitutiv ist. Die Pointe einer kritischen Gebetshermeneutik bestünde aber darin, gerade diesen Impuls aufzunehmen und die Frage zu stellen, an welchen Götzen unsere Gebete sich richten, oder anders gesagt: gerade die im Gebet errungene Einsicht, dass wir nicht beten können, ernst zu nehmen und damit mit dem Gebet als »erste[m] und grundlegende[m] Akt theologischer Arbeit ${ }^{49}$ ernst $\mathrm{zu}$ machen. In diesem Horizont scheint mir die Theologie in Jean-Louis Chrétien einen bislang unterschätzten Gesprächspartner finden zu können.

— Michael Braunschweig ist Assistent am Institut für Sozialethik der Universität Zürich.

\footnotetext{
${ }^{47}$ Vgl. ebd.

${ }^{48}$ Vgl. nochmals Chrétien, La parole blessée, 43.

${ }^{49}$ Karl Barth, Einführung in die evangelische Theologie, Zürich 1962, 176 (kursiv MUB).
} 